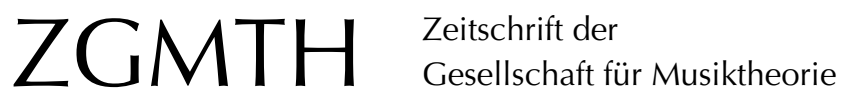

Menke, Johannes (2011): Die Familie der cadenza doppia. ZGMTH 8/3, 389-405.

https://doi.org/10.31751/654

(C) 2011 Johannes Menke

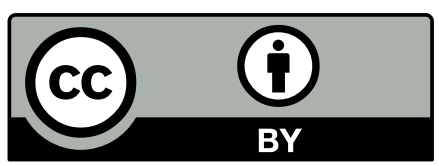

Dieser Text erscheint im Open Access und ist lizenziert unter einer Creative Commons Namensnennung 4.0 International Lizenz.

This is an open access article licensed under a

Creative Commons Attribution 4.0 International License.

veröffentlicht / first published: 29/03/2012

zuletzt geändert / last updated: 02/07/2013 


\title{
Die Familie der cadenza doppia ${ }^{1}$
}

\author{
Johannes Menke
}

ABSTRACT: Der Beitrag diskutiert die in der italienischen Theorie des 18. Jahrhunderts als cadenza doppia bezeichnete Kadenzformel und deren Ursprünge in der Theorie und Musik seit dem 15./16. Jahrhundert. Dabei zeigt sich, dass die von Vicentino bereits als altmodisch bezeichnete Formel ein reichhaltiges Potenzial für Variantenbildungen und Sequenzierungen aufweist, das im Laufe des 17. und 18. Jahrhunderts immer weiter entfaltet wird: Klauseln können variiert und getauscht, und der Bass kann diminuiert werden. Die Durchdringung des Satzes mit Klauselprogressionen der cadenza doppia trägt dabei maßgeblich zur Ausformulierung tonaler Klangfolgen bei. Die archaisch anmutenden Grundformen der Kadenzformel prägen die Musiksprache bis weit in die Romantik hinein.

\section{Vorbemerkung}

Tonale Satzmodelle, verstanden als "standardisierte musikalische Wendungen ${ }^{2}$, lassen sich hinsichtlich ihrer formalen Verwendung idealtypisch in Initial-, Sequenz- und Kadenzmodelle unterteilen. Obwohl sich die meisten bis ins 16., manchmal sogar ins späte 14. Jahrhundert zurückverfolgen lassen (wie etwa die 7-6-Consecutive) ${ }^{3}$, verfestigen sich viele Modelle im Generalbasszeitalter und werden nun 'greifbar in der Konfiguration von modellhaftem Bass, Generalbassbezifferung und paradigmatischem Oberstimmenverlauf. ${ }^{4}$

So plausibel der skizzierte Zugriff auf Satzmodelle erscheint und so sehr ich selbst ihn nach wie vor unterstütze, so dringend bedarf er andererseits der Ergänzung, Ausdifferenzierung und - wenn nötig - der Problematisierung. Nicht immer nämlich bilden die Koordinaten realer Bass/Generalbassziffer/Oberstimmenverlauf eine absolut fixe, ontische Konfiguration aus. Die Faszination der Satzmodelle liegt vielmehr darin begründet, dass sie einerseits bestimmbar und damit wiedererkennbar sind, andererseits entweder kombinierbar oder selbst aus kombinierbaren Bestandteilen zusammengesetzt sind: So

1 Dieser Text ist die schriftliche Fassung eines Vortrages, den ich am 21. März 2011 in Basel auf einem Symposium zum Thema »Satzmodelle des Generalbasszeitalters» gehalten habe.

2 Vgl. Schwab-Felisch 2010, 415.

3 Hierzu Jans 1987, Dodds 2006, Froebe 2009.

4 Die umfassendste mir bekannte Zusammenstellung von Generalbassmodellen ist das von Hans Peter Weber seit 1997 zusammengestellte und in seiner Art einzigartige Generalbass-Compendium, das der Autor in mehreren Auflagen als Unterrichtsmaterial für den Gehörbildungsunterricht an der Schola Cantorum Basiliensis zusammengestellt hat (Weber 2004). Leider ist diese Schrift nicht im Handel erhältlich und daher in Fachkreisen der Musiktheorie kaum bekannt, weshalb ich in diesem Zusammenhang unbedingt auf diese Schrift hinweisen möchte. 
lässt sich die bereits erwähnte 7-6-Consecutive bekanntlich mit einem Quintfall und, wird sie in den Oberstimmen nur zweistimmig ausgeführt, mit nahezu sämtlichen Basssequenzen kombinieren. ${ }^{5}$

Es lassen sich zuweilen regelrechte `Familienverbände` ausfindig machen, die man weder durch eine Rückführung auf reale Bässe, Fundamentalbässe oder andere Strukturschemata ohne weiteres systematisieren kann. Auch ihre jeweilige Verwendung in bestimmten formalen Situationen lässt sich nicht auf einen Nenner bringen. Gleichzeitig vermögen solche Familien aufgrund ihrer Langlebigkeit und ihrer weiten Verzweigung die interessantesten Angehörigen hervorzubringen. Um eine solche Familie soll es im Folgenden gehen. Unter Verwendung desjenigen Begriffes, der einem zentralen Familienmitglied für längere Zeit anhaftete, könnte man sie die Familie der cadenza doppia nennen. Im Folgenden sollen der Variantenreichtum dieses Modells und die Vielfalt seiner formalen Kontextualisierungen erörtert werden.

\section{Geschichte, Terminologie und Systematik der cadenza doppia}

Die erste mir bekannte Quelle, in der das Phänomen der cadenza doppia beschrieben wird, ist Nicola Vicentinos L'antica musica ridotta alla moderna prattica (Rom 1555). In einer Beispielsammlung zur Behandlung der synkopierten Quarte teilt Vicentino im letzten Kasten eine Kadenz mit synkopierter Quarte über der liegenden Bassklauselpenultima mit, die er »all' antica« überschreibt. Im zughörigen Textteil wird diese Form der Quartdissonanz als »sincopa tutta cattiva» bezeichnet (übersetzt »die ganz falsche Synkope«), die nicht mehr zeitgemäß sei (»non è moderna«). ${ }^{6}$

\section{Ef empio della Qutrta fincopata à duc uvei, ¿ non fincop.à tre uoci, ¿ con il punto : come s'tia da comporre à molte uoci, \& con la fincopa tutte cattina.}

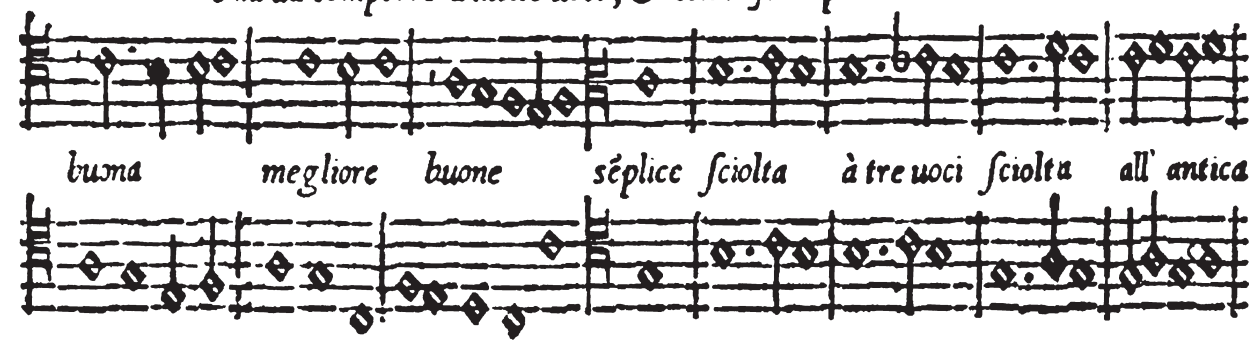

I.

2.

3.

Si fude legare piu quarte, con le terze difcendents, fingendo di uoler finire la fincopa; bora nel primo, et bora nel fes

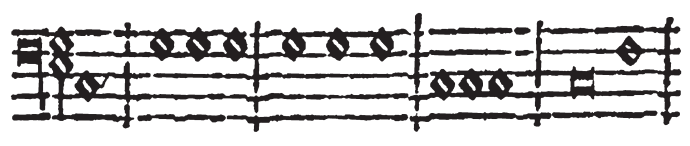

Beispiel 1: Vicentino 1555, 30

5 Vgl. hierzu Menke 2009, 102.

6 Vicentino 1555, 30. 
Über dem `dominantischen` Penultima-Basston c lassen sich die alternativen Oberstimmen in Generalbassbezifferungen ${ }^{7}$ folgendermaßen darstellen:

\section{5}

3443

Es handelt sich also um ein nach vorne erweitertes Gerüst aus Diskant- (3443) und Tenorklausel (5655). ${ }^{8}$ Dieselbe Formel wird in einer Vielzahl italienischer Quellen des 18. Jahrhunderts als cadenza doppia bezeichnet und bildet in dieser Ausprägung den Ausgangspunkt der Untersuchung.

Die Einschätzung Vicentinos, die von ihm als »all'antica" qualifizierte Kadenzformel sei veraltet, ist angesichts ihres inflationären Gebrauchs im Frottola-Repertoire durchaus nachvollziehbar. Denn dieses entstammt, obgleich es erst im frühen 16. Jahrhundert gedruckt wurde, ganz überwiegend dem späten 15. Jahrhundert. Das folgende Beispiel zeigt eine für dieses Repertoire typische Häufung der entsprechenden Formel. So finden wir im zweiten Takt des Beispiels diese Kombination:

5555

3443

Im vierten Takt:

5455

3443

Im vorletzten Takt schließlich erscheinen zwar die für die cadenza doppia typische Diskant- und Tenorklausel, allerdings kombiniert mit einem variierten Bassverlauf (Bassstufen 5-6-5). Bereits hier wird evident, dass es möglich ist, den Bass zu verändern - ein Verfahren, das im 17. Jahrhundert reiche Blüten hervorbringen wird.

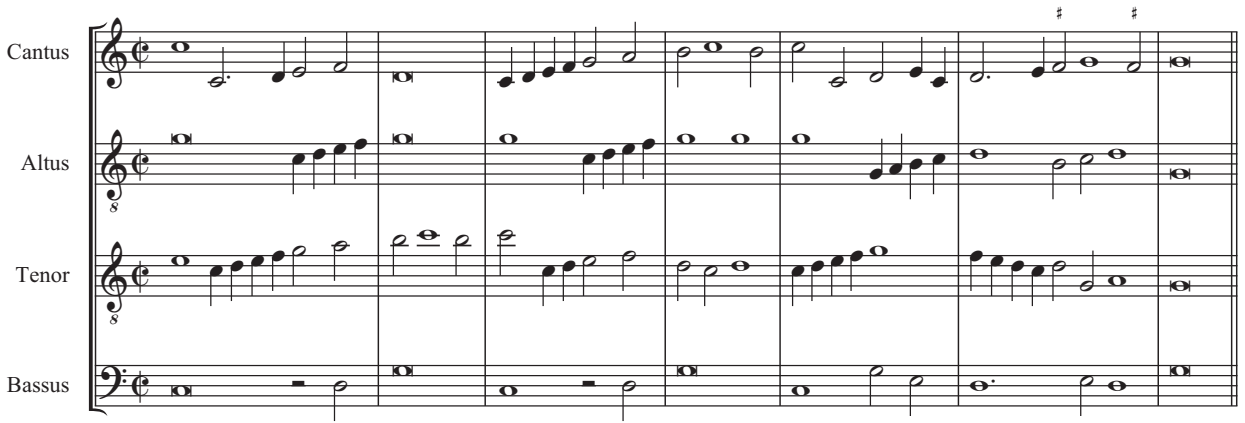

Beispiel 2: Ottaviano Petrucci, Strambotti, Ode, Frottole, libro quarto (Venedig 1505), "Gli angelici sembianti«

7 Um 1555 wurde der Generalbass womöglich bereits praktiziert, Notenausgaben mit Generalbassstimmen lassen sich seit den 1580er Jahren nachweisen.

8 Vgl. grundsätzlich zu Kadenz und Klausel Holtmeier 2010. 
In der Tat handelt es sich hier um eine zu Vicentinos Zeiten etablierte Formel, die bereits in verschiedenen Varianten verwendet wird und ein genuin italienischer Beitrag zu sein scheint: Man findet vergleichbare Wendungen bei den französischen und niederländischen Meistern eher selten, und wenn dann bei denjenigen, die - wie etwa Heinrich Isaac - lange Zeit in Italien gelebt haben.

Was die Modernität der von Vicentino als all'antica bezeichneten Kadenzformel angeht, sollte dieser sich in Hinblick auf ihre Zukunft allerdings gründlich geirrt haben: Die ihm nachfolgende Generation verwendet diese Formel immer häufiger, und das 17. Jahrhundert ist, wie wir sehen werden, von ihr geradezu besessen. Doch bleiben wir zunächst im 16. Jahrhundert. Gioseffo Zarlino zeigt in den Istitutioni harmoniche (Venedig 1558) eine ähnliche Kadenzformel im Kapitel über den allgemeinen Gebrauch der Quarte, ohne jedoch auf die Kadenzformel als solche einzugehen oder ihr einen eigenen Begriff zuzuweisen.

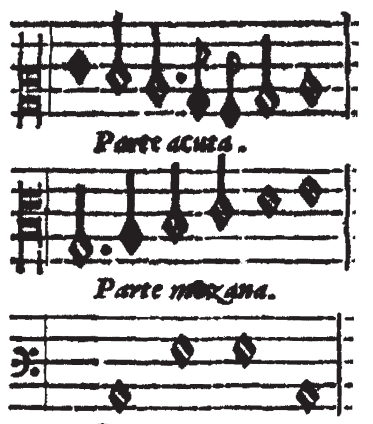

Parte grave.

Zarlino führt die Formel in einer speziellen Variante, nämlich mit einer Stimmkreuzung, vor:

\section{3}

3455

Versucht man alle seit dem 16. Jahrhundert gebräuchlichen Varianten zusammenzufassen, so könnte man - ohne Berücksichtigung der Bass-Varianten - zu folgendem Resultat gelangen:

\begin{tabular}{|l|l|l|l|l|l|l|l|}
\hline a) & b) & c) & d) & e) & f) & g) & h) \\
\hline 5455 & 5443 & 5555 & 5655 & 6655 & 6655 & 7655 & 7657 \\
\hline 3443 & 3455 & 3443 & 3443 & 3443 & 5443 & 3443 & 3443 \\
\hline
\end{tabular}

Die Übersicht macht deutlich, dass mit Ausnahme von b) und f) nur die Tenorklauseln variiert werden, während die Diskantklauseln gleich bleiben. Das konstituierende Merkmal ist - auch bei Variante b) - die quarta consonans auf der zweiten Zählzeit, der eine quarta dissonans auf der dritten Zählzeit unmittelbar folgt. In allen Varianten entsteht an dieser Stelle ein Quart-Quintklang. Es handelt sich also um eine spezielle Art der Syn- 
kopendissonanz, bei der dasselbe Intervall - die Quarte - zunächst als konsonant, dann als dissonant aufgefasst wird. Daraus resultieren die Klänge ,Doppelquarte ‘ bei a) und b), 'Quartquint ‘ bei c) und 'Quartsext ( bei d), e) und f), die eigentlich dissonant sind, in diesem Kontext aber behandelt werden, als wären sie konsonant. Wie im nächsten Abschnitt gezeigt wird, entspringen daraus bei gleichzeitiger Bassvariation bestimmte Formen der scheinbar sfreien Behandlung dissonanter Klänge.

Der Begriffsgebrauch vor dem 18. Jahrhundert ist uneinheitlich. Um nur einige Stichproben herauszugreifen: Die von Vicentino verwendeten Termini der sincopa tutta cattiva sowie der all'antica verweisen auf die Dissonanzhaltigkeit bzw. auf den von ihm konstatierten saltmodischen< Charakter des Modells. Bei Zarlino findet sich kein Begriff, ebenso wenig später bei Lorenzo Penna (1684). ${ }^{9}$ In der Kadenzordnung Georg Muffats (1699) taucht die Formel als »Gantze Cadenz oder Cadentia major perfectis « auf..$^{10}$ Damit weist er sie als ıvollständige Version der "Cadentia major« aus, die in Muffats ganz klassischer Kadenztypologie den hierarchisch obersten Rang einnimmt. ${ }^{11}$ Damit ist die "Cadentia major perfectis" die stärkste Kadenz überhaupt - eine Rolle, die sie auch in der Theorie des 18. Jahrhundert behalten wird. ${ }^{12}$ In seinem Musicalische[n] Lexicon (1732) bezeichnet Johann Gottfried Walther sie als "Cadenza composta maggiore « ${ }^{13}$, und verwendet damit einem italienischen Terminus, den er vermutlich von Franceso Gasparini übernommen hat. ${ }^{14}$ In den neapolitanischen Konservatorien des 18. und 19. Jahrhunderts setzt sich schließlich der Terminus der cadenza doppia durch, den man bei Franceso Durante, Fedele Fenaroli, Giacomo Tritto, Giovanni Furno und zahlreichen anderen Lehrern findet. ${ }^{15}$

\section{Abgeleitete Formen}

Francesco Gasparini stellt zusammen mit der cadenza composta maggiore, der späteren cadenza doppia, auch mehrere Möglichkeiten vor, die Formel zu diminuieren:

Die diminuierten Kadenzen (Cadenze diminuite) sind vielgestaltig, und sie leiten sich her von der oben erwähnten Cadenza maggiore und minore, weil die Note, welche die Kadenz formt [gemeint ist der Bass], in zwei, vier, oder mehr Noten geteilt wird. ${ }^{16}$

9 Penna 1684, 173.

10 Muffat 1699, 106.

11 Die Cadentia major hat den Quintfall im Bass (Stufenfolge 5-1), die Cadentia minor den Quartfall (Stufenfolge 4-1 oder 1-5), die Cadentia minima den absteigenden Sekundschritt (Stufenfolge 2-1 oder 6-5).

12 In der Praxis setzen sich, zumal in der Instrumentalmusik, die cadenza composta und cadenza semplice als Standards durch, so dass der cadenza doppia spätestens in der zweiten Jahrhunderthälfte ein altertümlicher Charakter zugewachsen ist, auf den ich später noch zu sprechen kommen werde.

13 Walther 1732, 124.

14 Vgl. Gasparini 1722, 31.

15 Vgl. Durante 2003, 5; Fenaroli 1775, 7 f.; Tritto 1816, 19; Furno 1817.

16 Übersetzung d. Verf., Gasparini 1722, 31: „Le Cadenze diminuite sono diverse, e derivano dalle sopradette maggiori, e minori, poiche la nota, che forma la Cadenza sarà divisa in due, o sia in quattro, o più note." 


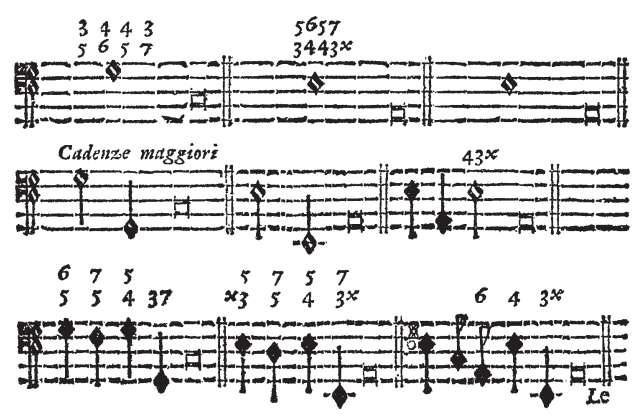

Beispiel 4: Gasparini 1722, 31, Cadenze diminuite

Diese Technik ist von kaum zu unterschätzender Bedeutung für die Satztechnik des 17. und frühen 18. Jahrhunderts. Ich möchte dies an nur einem Beispiel erläutern: Friedrich Erhard Niedt gibt in seiner Musicalischen Handleitung einen »schlechten«, also ıschlichten Bass, auf dessen Grundlage er exemplarische Suitensätze komponiert. ${ }^{17}$ In diesem "schlechten « Bass gibt es vier Kadenzen, bei denen es sich ausnahmslos um cadenze doppie handelt. In der Ausformung der jeweiligen Suitensätze wird es allerdings nötig, diese basalen Modelle durch individuelle diminuierte Formen zu ersetzen. Ein Blick in ausgewählte Kompositionen Chambonnieres', D'Angleberts oder auch noch Händels zeigt, dass das Verfahren der cadenza doppia diminuita offenbar über lange Zeit angewandt wurde.

Die Vielzahl der möglichen Bassdiminutionen lässt sich nur schwer überblicken. Es stellt sich jedoch die Frage, welche genuinen Klangfolgen aus diesen Möglichkeiten resultieren können.

In den Beispielen Gasparinis (Beispiel 4) bleibt die Kadenzfunktion zweifelsohne erhalten, jedoch entstehen auf der zweiten Zählzeit zweimal Septakkorde auf der vierten Stufe, deren Septime in einem Fall nicht vorbereitet ist. Eine solche unvorbereitete Septime ist nicht generell typisch für den Gebrauch der Septime im barocken Kontrapunkt, wohl aber im Kadenzrahmen. Die folgende Übersicht zeigt Klangfolgen, die aus drei verschiedenen Oberstimmenführungen resultieren können (Beispiel 5).

Den Ausgangspunkt der Darstellung bilden nur drei mögliche Oberstimmenverläufe. Unterlegt sind ihnen die wichtigsten der möglichen Bassverläufe; vor allem durch ornamentale Bassdiminution kann eine Vielzahl weiterer Varianten gewonnen werden. Viele der resultierenden Klangfolgen sind unproblematisch, einige aber erfordern eine gesonderte Betrachtung:

In den Konstellationen 1b, 1d sowie 1e ergibt sich ein unvorbereiteter Septakkord auf der vierten Stufe. ${ }^{18}$ Dies wiegt im letzteren Fall (1e) umso schwerer, als der davor befindliche Sekundakkord nicht normal aufgelöst wird; handelt es sich bei der abwärts

17 Niedt 1710-21, 117.

18 Bei Bartolomeo Bismantova findet sich die Variante 1b als »Preparamento alla Cadenza« (Bismantova 1677,80$)$. Womöglich lässt sich auch das erste von Bismantova angeführte »Preparamento alla Cadenza«, das in der Bassführung und der freien Behandlung des Terzquartakkordes auf der sechsten Stufe an Christoph Bernhards Cadentia duriuscula erinnert, als entfernte Verwandte der cadenza doppia begreifen. 
1)

2)

3)

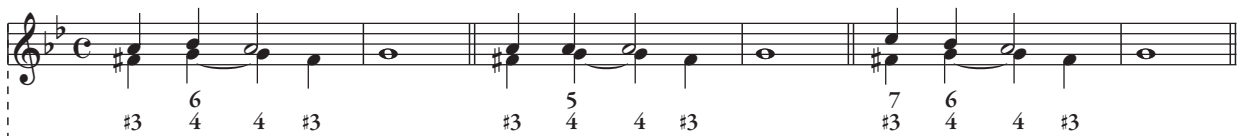

a)

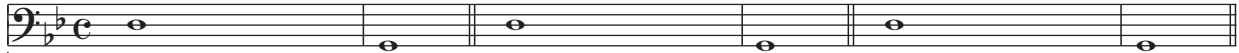

b)

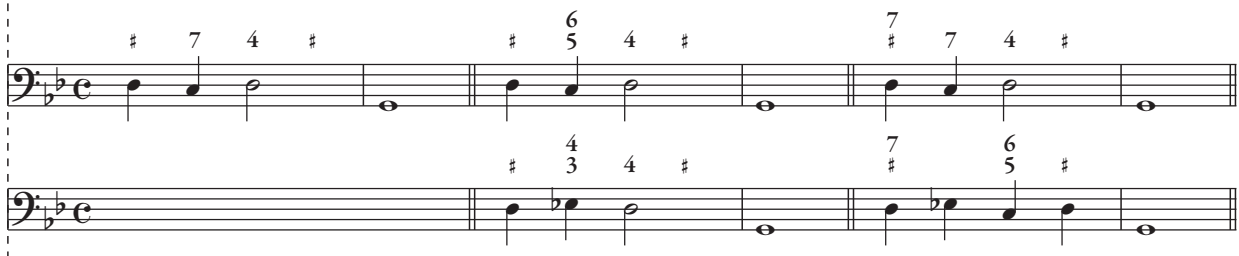

d)

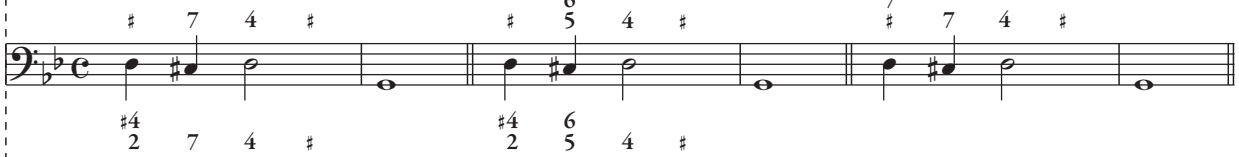

e)
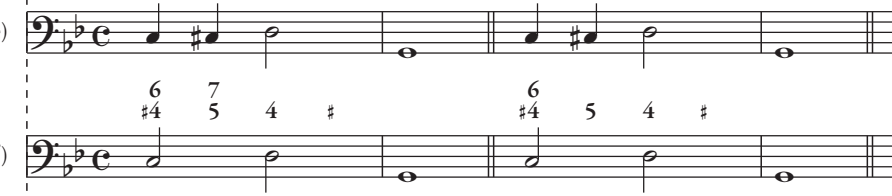

g) $\begin{array}{lll} & 6 & \end{array}$

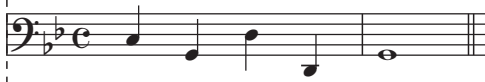

h)

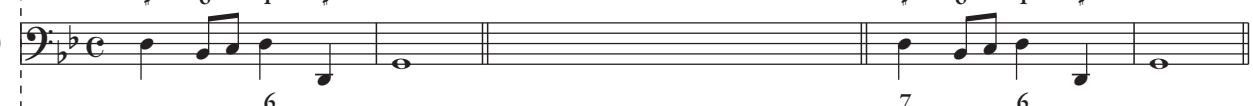

i)

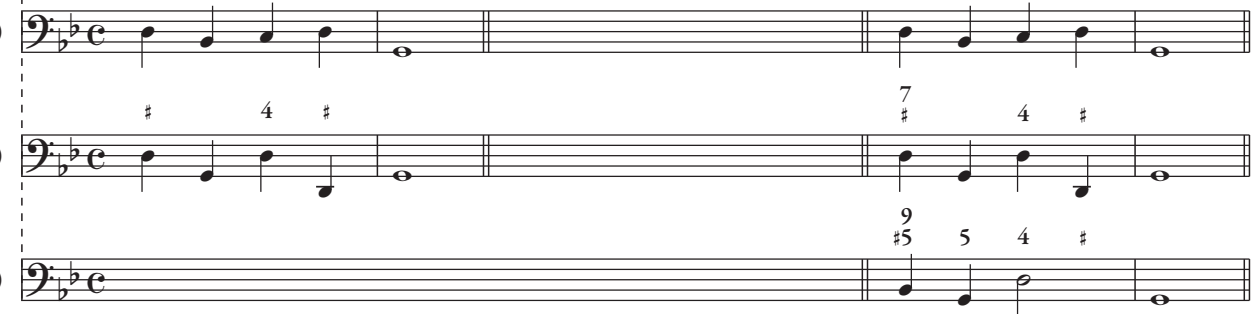

Beispiel 5: Klangfolgen mollarer Kadenzen, die aus einer Bassdiminution der cadenza doppia resultieren können

aufzulösenden Dissonanz doch eigentlich um den Basston. Die so entstehende Klangprogression hat gerade durch die zugleich überraschende und formelhaft rabgesichertes Weiterführung des Sekundakkordes in den verminderten Septakkord über der chromatisch erhöhten vierten Stufe eine eigentümlich dramatische Wirkung. Diese wird von den 
Komponisten auch entsprechend wirkungsvoll eingesetzt - man denke etwa an die Takte 9 bis 11 des ersten Satzes aus Antonio Vivaldis "L'inverno « aus Le quattro stagioni, wo die für Vivaldi so charakteristischen Akkordwiederholungen einen entsprechend insistierenden Effekt hervorrufen. Eine abgemilderte Variante dieser Progression ist 2e. In den Kombinationen 3b und 3d löst sich die Septime über der fünften Stufe der ersten Zählzeit direkt in die Septime der vierten Stufe auf. Diese Situation wird auch in der zeitgenössischen Theorie reflektiert, etwa bei Playford ${ }^{19}$ und Bononcini. ${ }^{20}$ Bei Corelli lässt sich die Wendung ab op. 2 sehr häufig beobachten.

Der unvorbereitete Quintsextakkord der Varianten 2b und 2e gehört stilistisch der Musik des mittleren 17. Jahrhunderts an, wie ein kurzer Auszug aus Giacomo Carissimis Jonas veranschaulichen mag, wo die entsprechende Klangfolge der Eindringlichkeit halber eine Quarte höher wiederholt wird.

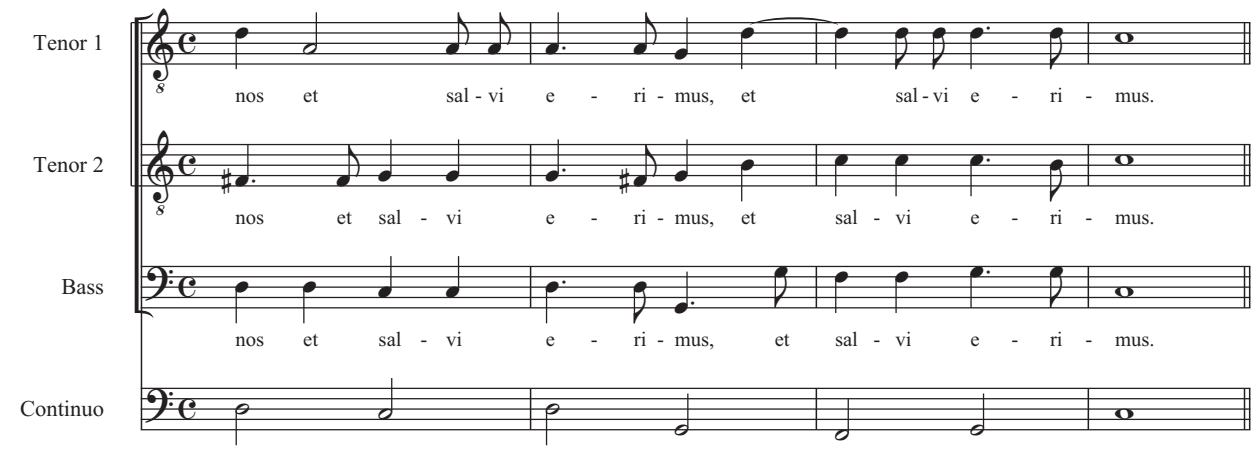

Beispiel 6: Giacomo Carissimi, Jonas, Ende des Chores »Dii magni«

Auf eine ähnliche Weise kommt der Terzquartakkord auf der sechsten Stufe bei 2c zustande. Die im Terzquartakkord aufgrund ihrer Sekundreibung zur Quarte dissonante Terz erscheint hier unvorbereitet. Georg Muffat subsumiert diese Konstellation unter die Rubrik »Quarta Italica«:

Quarta Italica, die welsche oder irregular Quart, welche etwas seltzamer vorkombt, ist ein Grieff, in welchen, da der Baß sich beweget, in einer obern Stirne aber die Quart vor und nach fest haltet, mit welcher Quart die Terz in dreystimmiger Harmoni vor und nach gradatim steigend genomen wird, Exempl A. ${ }^{21}$

19 Vgl. Playford 1697, 86.

20 Vgl. Bononcini 1673, 68.

21 Muffat 1699, 16. Muffats Klassifizierung der seinerzeit modernen Verwendung der Quarte bietet einen exemplarischen Einblick in die Vielfalt der hochbarocken Klangsprache. Die Entstehung der quarta italica im Kadenzusammenhang ist für Muffat, wie seine Übersicht auf S. 21 zeigt, zwar nur eine ihrer Erscheinungsformen, dafür aber die für ihn repräsentativste. 


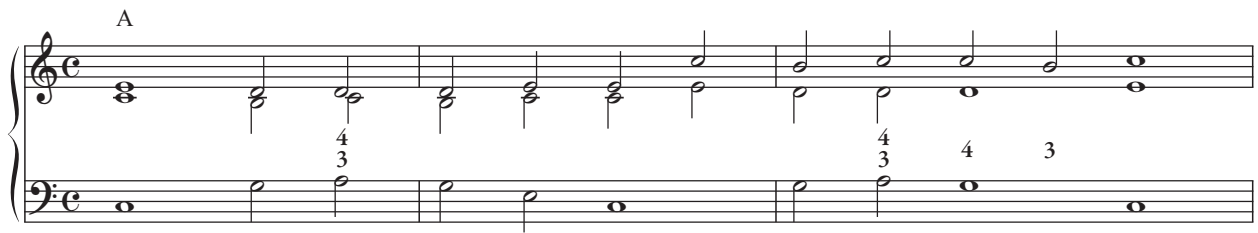

Beispiel 7: Muffat 1699, 16, Beispiel zur quarta italica

Zuletzt sei noch die Version 3k hervorgehoben: Hier entsteht auf der ersten Zählzeit ein Akkord mit übermäßiger Quinte und None auf der dritten Stufe, der charakteristisch für den französischen Stil ist und bei Jean-François Dandrieu als »Quinte Superflue» bezeichnet wird. ${ }^{22}$ Ein Beispiel für diese Variante findet sich etwa in der Messe de Minuit von Marc-Antoine Charpentier:

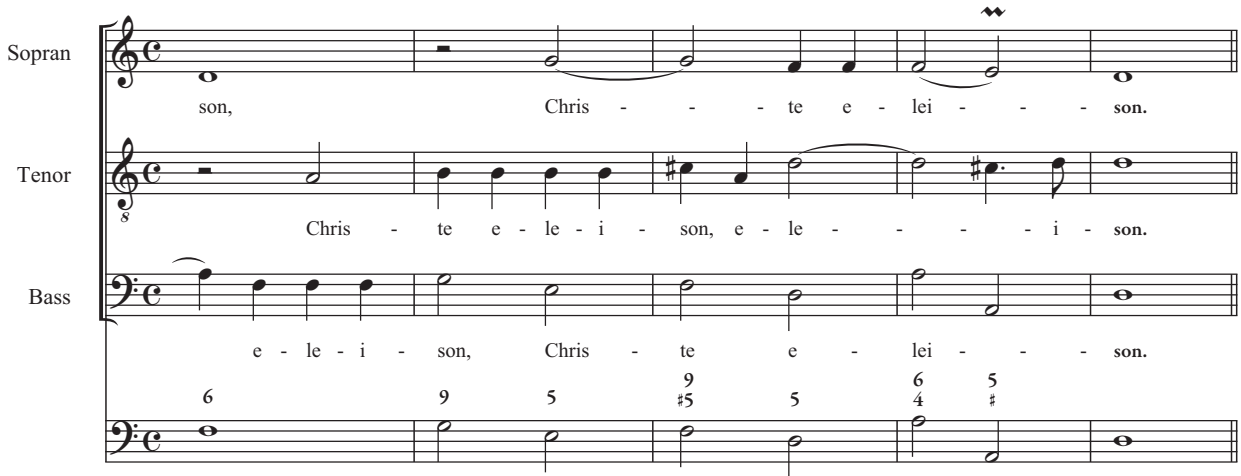

Beispiel 8: Marc-Antoine Charpentier, Messe de Minuit, Christe, T, 70-74

\section{Varianten ohne syntaktische Kadenzfunktion}

In allen bislang besprochenen Varianten der cadenza doppia blieb ihre syntaktische Funktion als Kadenz im Sinne eines definitiven Phrasenendes erhalten. Unter den vielfältigen kontrapunktischen Möglichkeiten der Kadenzflucht gibt es zwei Techniken, die nicht erst zum Eintritt der Ultima (wie beim Trugschluss), sondern bereits innerhalb der Kadenz eine kadenzgemäße perfectio verhindern: Jeweils auf der letzten Zählzeit der Penultima verhindert entweder eine Mollterz in der betroffenen Oberstimme (a) oder ein Schritt in die vierte Bassstufe mit darüber befindlichem Tritonusakkord ${ }^{23}$ (b) ein Fortschreiten in die Finalis.

22 Dandrieu 1719, Tafel XIV. Bei Dandrieu befindet sich dieser Klang stets auf der dritten Bassstufe in Moll und bewegt sich - wie in unserer Kadenzwendung - in die erste weiter, um die Auflösung der übermäßigen Quinte zu ermöglichen.

23 Der Terminus »Tritonusakkord« beschreibt, der französischen Tradition folgend (»l'Acord du Triton«), den Sekundakkord auf der schrittweise abwärts gehenden vierten Stufe mit der für diese Situation charakteristischen übermäßigen Quarte (Tritonus). 


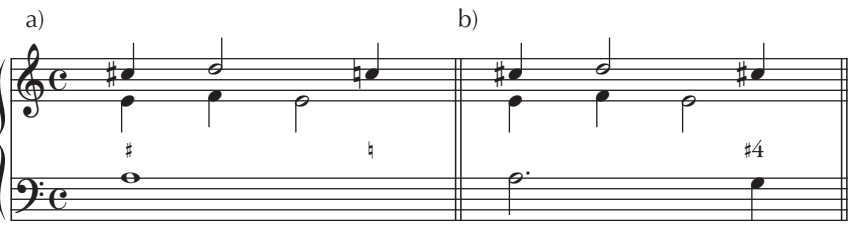

Beispiel 9: Kadenzimperfektion durch Mollterz (a) oder Tritonusakkord (b)

\section{Die cadenza doppia als Initialmodell}

Obwohl die cadenza doppia per definitionem als Endigungsformel ausgewiesen ist, lässt sie sich ebenso als Initialformel finden. Ein Beispiel bietet der Beginn der Sonata op. 4,3 von Azzolino Bernardino della Ciaja:

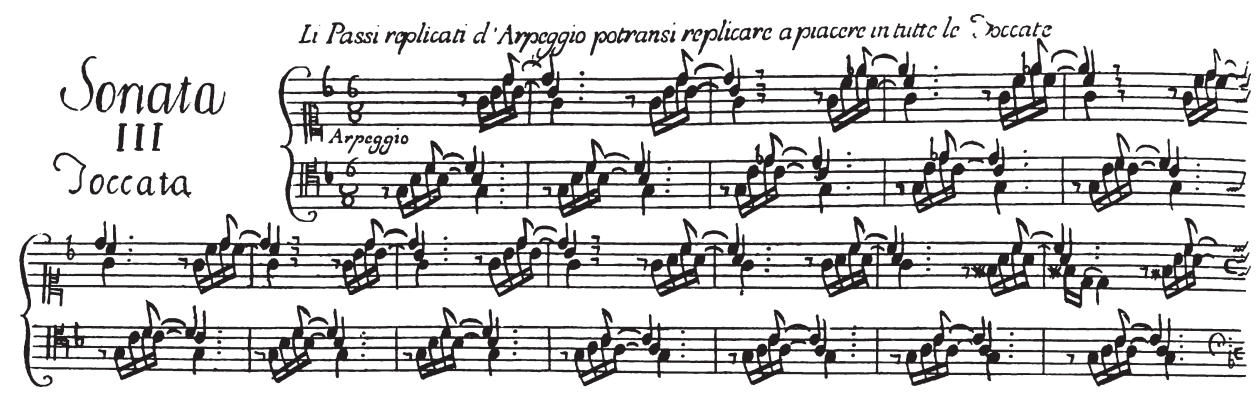

Beispiel 10: Azzolino Bernardino della Ciaja, Sonata op. 4,3 (Rom 1727), Anfang des I. Satzes mit cadenza doppia als Initialmodell

Über dem Orgelpunkt $g$ erscheinen hier quasi in Zeitlupe die Klänge der cadenza doppia nacheinander: Terzquint, Quartsext, Quartquint, Terzquint.

Es lassen sich leicht mehr Beispiele anführen; um ins französische Repertoire zu wechseln, etwa die Allemande Nr. 19 aus dem Livre Premier von Jacques Champion de Chambonnières (1670) oder die Allemande der ersten Suite aus den Pieces de Clavessin von Jean-Henry d'Anglebert (1689).

\section{Sequenzierung}

Angelo Berardi gibt in seinen Documenti armonici (Bologna 1687) unter der Überschrift »Motivo di cadenza« ein Beispiel für die Sequenzierung der cadenza doppia.

Hierbei wird durch das Eintreten der Mollterz auf der zweiten Takthälfte in Takt 3 die Kadenzwirkung außer Kraft gesetzt und gleichzeitig die Septime im nächsten Takt vorbereitet. Im Folgenden wechseln in den Oberstimmen die Diskantklausel 3443 und die Tenorklausel 7655 einander ab, der Bass schreitet in fallenden Quinten/steigenden 


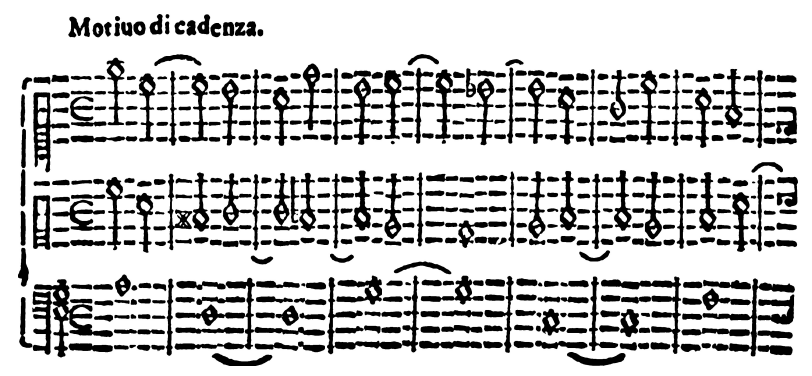

Beispiel 11: Berardi 1687, 151, »Motivo di cadenza«

Quarten fort. Franz Xaver Murschhauser gibt 1721 eine anschauliche Beschreibung dieses Verfahrens:

»Es gibt neben diesen auch noch eine andere Gattung der Cadenzen / so Cadentiae imperfectae, oder unvollkommene Cadenzen benahmt werden / und sind diejenige / welche sich zwar dergestalten anbinden / als wollten sie eine Cadenz ausmachen; wann es aber zu den Schluß kommet / sich weiters bewegen / und die Modulation wiederum fortsetzen. ${ }^{24}$

Ulrich Kaiser und Andreas Helmberger zeigen, wie schon Heinrich Deppert, welche Möglichkeiten sich aus dieser Fortschreitung im 18. Jahrhundert ergeben, so dass eine weitere Ausführung an dieser Stelle nicht notwendig ist. ${ }^{25}$ Es sei hier lediglich der Weg skizziert, der zu Berardi führt. Ornithoparcus gibt 1517 einen interessanten Hinweis auf die Bedeutung der Klausel im Allgemeinen:

»Zehntens. Jeder Gesang ist süßer, wenn in ihm Standard-Klauseln [clausulis formalibus] im Überfluss vorhanden sind. In den Klauseln ist nämlich eine solche Kraft, dass sie wegen der perfectio ${ }^{26}$ sogar die Dissonanzen konsonant macht. $\aleph^{27}$

Ornithoparcus verwendet in seinem Beispiel zwar selbst keine cadenze doppie, sein Ideal omnipräsenter Klauseln lässt sich aber mithilfe der cadenza doppia trefflich realisieren. Eine entsprechend hohe Klauseldichte zeigt etwa die folgende Passage aus der Missa de Beata Virgine von Christóbal de Morales aus dem Jahr 1540, in der die cadenza doppia eine Quarte abwärts sequenziert wird:

24 Murschhauser 1721, 78.

25 Deppert 1993, 152-169. Helmberger/Kaiser o.J.: http://www.musiktheorie-aktuell.de/tutorials/ motivodicadenza.aspx.

26 Mit perfectio ist wie bei Johannes Tinctoris der jeder Kadenz innewohnende Prozess des KonsonantWerdens gemeint, exemplarisch das Spannungsgefälle Septime-Sexte-Oktave.

27 Übersetzung d. Verf.: »Decima, omnis cantilena eo est suauior, quo clausulis formalibus abundantior. Tanta enim clausulis inest vis, vt etiam dissonantias faciat consonas perfectionis gratia." (Ornithoparcus 1517, o.S.) 


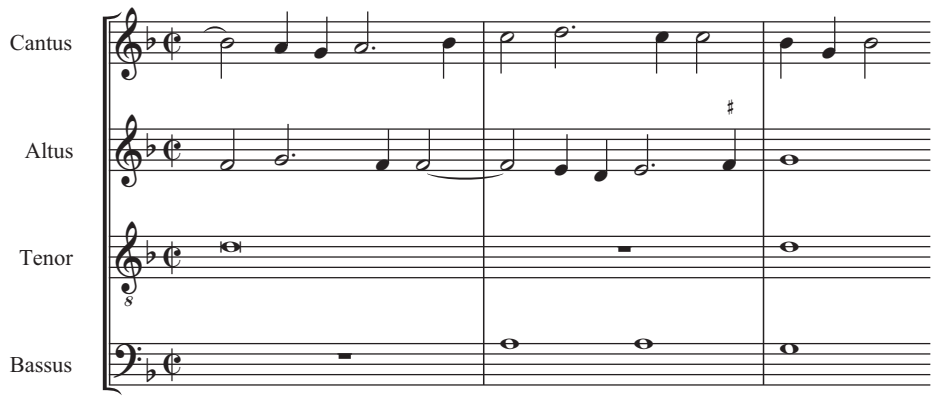

Beispiel 12: Christóbal de Morales, Missa de Beata Virgine (1540), Christe, T. 19 f.

Eine Sequenzierung der Klauselprogression um eine Quarte aufwärts findet sich beispielsweise im Christe der Missa Nasce la gioia mia von Giovanni Pierluigi da Palestrina:

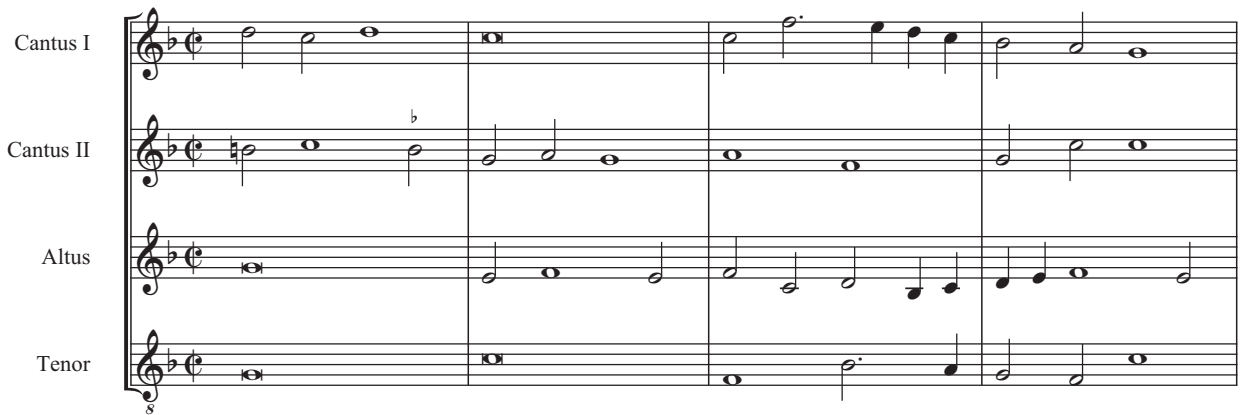

Beispiel 13: Pierluigi da Palestrina, Missa Nasce la gioia mia (1590), Christe, T. 27 f.

In Giovanni Maria Trabacis Toccata di durezze et legature von 1602 finden wir eine zweimalige Sequenzierung im Quintfall. Trabaci verwendet die Kombination aus der Diskantklausel 3443 und der Tenorklausel 6655, bei der unter der kleinen Sexte eine expressive verminderte Quarte entsteht:

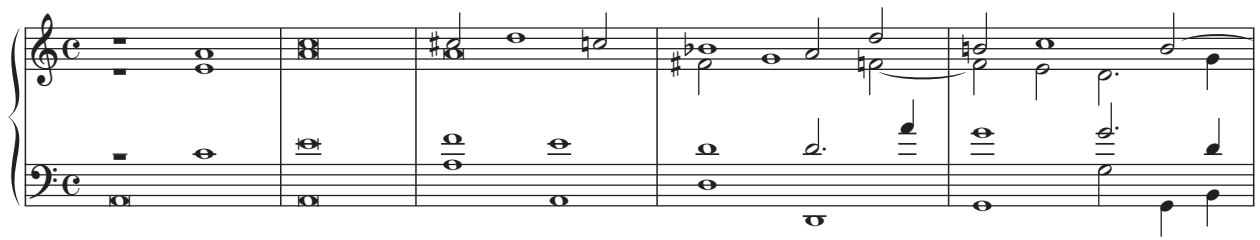

Beispiel 14: Giovanni Maria Trabaci, Componimenti per organo (1603), Toccata di durezze et legature, T. 1-5 
Im folgenden Solfeggiamento von Pompeo Natale schließlich finden wir sowohl die Sequenzierung um Sekunden aufwärts als auch um eine Terz abwärts. In diesen Fällen werden Diskant- und Tenorklausel nicht getauscht.

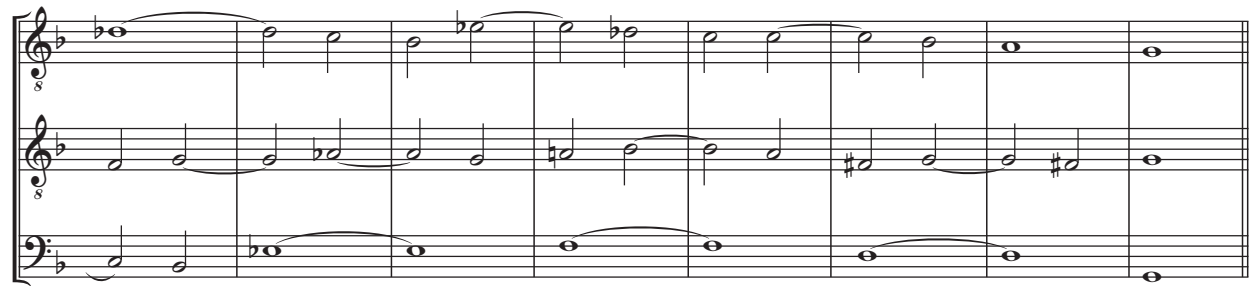

Beispiel 15: Pompeo Natale, Solfeggiamento XXXXV (1681), T. 14-21

Prinzipiell lässt sich die cadenza doppia um jedes Intervall sequenzieren. Dies eröffnet die Möglichkeit, einen im Bass befindlichen cantus firmus mit cadenze doppie zu begleiten - eine Praxis, die in Spanien verbreitet gewesen zu sein scheint. ${ }^{28}$ Am häufigsten sind allerdings die Sequenzierungen um fallende Quinten/steigende Quarten und um steigende Sekunden anzutreffen.

Wie Deppert, Helmberger und Kaiser gezeigt haben, lassen sich die Bässe diminuieren und die Klauseln vertauschen. Dieses Verfahren wendet beispielsweise Johann Kaspar Kerll in seiner Toccata 1 in den Takten 40-44 an. ${ }^{29}$ Aus dem Wohltemperierten Klavier seien folgende Passagen genannt: Es-Dur Präludium (T. 15 ff.) aus dem WTK I, Es-Dur-Fuge (T. 50 ff.) oder die As-Dur Fuge (T. 20 ff.) aus dem WTK II.

Vor allem der Klauseltausch verschleiert den Sequenzverlauf und verleiht den entsprechenden Passagen den Anschein eines assoziativen Spiels mit den Klauseln der cadenza doppia. Die sfreie Verkettung von Klauselkomplexen ohne übergeordneten Sequenzverlauf ist tatsächlich eine gängige Kompositionsmethode seit dem 17. Jahrhundert. Im folgenden Beispiel von Samuel Scheidt werden fast ausschließlich Diskant- und Tenorklauseln der cadenza doppia miteinander kombiniert, wobei die Diskantklausel durchweg eine die Kadenz flüchtende chromatische Veränderung des Leittones aufweist und die Tenorklausel in einer durch eine eingeschobene Bassklausel figurierten Variante verwendet wird.

28 Diesen Hinweis verdanke ich meinem Kollegen David Mesquita.

29 Johann Kaspar Kerll, Sämtliche Werke für Tasteninstrumente Bd. II, UE Wien 1991. 
$\longrightarrow$ Doppia-Sopranklausel

Doppia-Tenorklausel
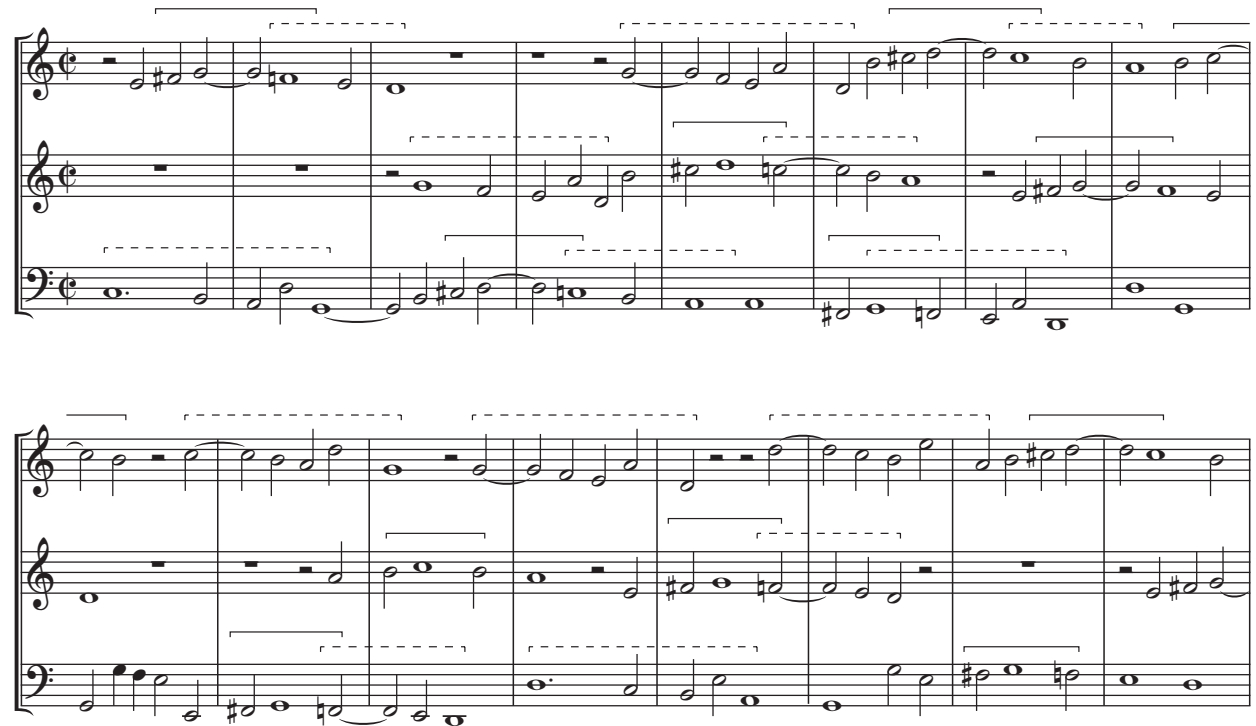

Beispiel 16: Samuel Scheidt, Symphonia Nr. 1 (1644), Komposition mit den Klauseln der cadenza doppia

Wie Ludwig Holtmeier gezeigt hat, lässt sich auch die nach 1700 kodifizierte Oktavregel als Ineinandergreifen von doppia-Klauseln begreifen: ${ }^{30}$

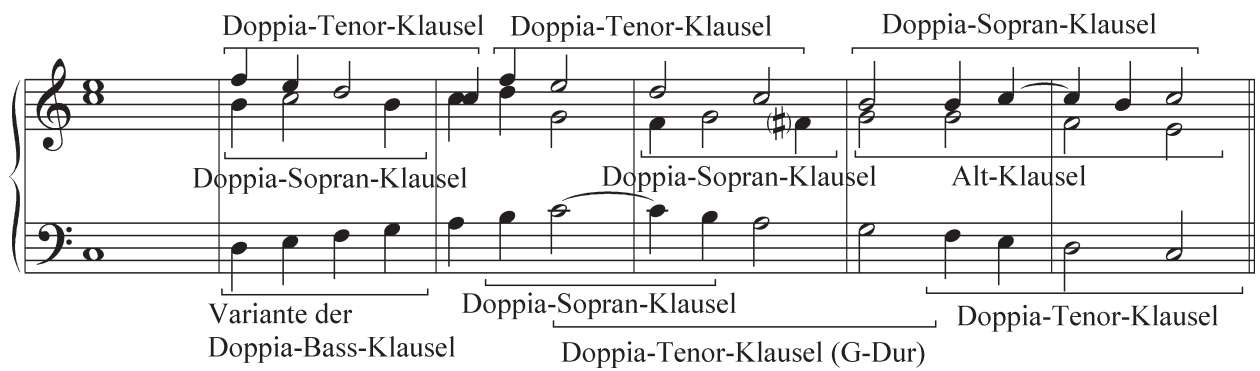

Beispiel 17: Oktavregel als Kombination von Klauseln aus der cadenza doppia ${ }^{31}$

Während somit die cadenza doppia in der Oktavregel gleichsam saufgehoben s ist, lebt sie als isolierte Kadenzformel gleichwohl weiter. Ein Blick in Beethovens »Mondschein-

30 Vgl. Holtmeier 2008, 14.

31 Aus: Paisiello, 2008, 152. 
sonate« (op. 27, Nr. 2) etwa zeigt, dass die sarchaische` Endigungsformel dort (T. 4) keineswegs in Widerspruch zu Beethovens Tonsprache steht, sondern durch ihre gleichsam archaische Aura den spezifisch ‘romantischen`Gestus des Stückes an dieser Stelle maßgeblich prägt.

Zum Archaischen der cadenza doppia in der Romantik gehört die charakteristische quarta consonans, die sich - wie die eingangs präsentierte Systematik verdeutlicht - in allen Ausprägungen findet, die auf der Bassklausel basieren. So gesehen, könnte man auch den berühmten "Scheidegesang" (Wolzogen) aus Richard Wagners Tristan und Isolde als eine späte Ausprägung der cadenza doppia interpretieren. Um diese vielleicht ungewohnte Perspektive zu verdeutlichen, zeigt das folgende Beispiel eine Generalbassreduktion der Passage. Die anfangs etablierte melodische Formel (1443 über dem Bass) wird ab Takt 6 über der Tenorklausel im Bass sequenziell fortgesetzt.

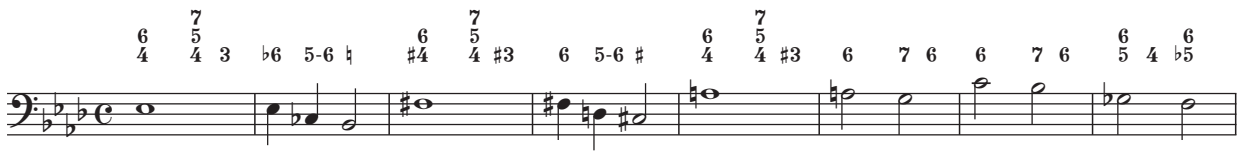

Beispiel 18: Richard Wagner, Tristan und Isolde, »Mild und leise«, Dritter Aufzug, Szene 3 in Generalbassreduktion

\section{Fazit}

Ausgehend von standardisierenden Endigungsformeln des Frottola-Repertoires mit quarta consonans lässt sich beobachten, wie sich seit dem späten 15. bzw. frühen 16. Jahrhundert eine ganze `Familie` von Kadenzformeln entwickelt, deren `Angehörige` durch Techniken der Variation, der Diminution, des Stimmtauschs und der Sequenzierung entstehen. Auch wenn die cadenza doppia eine genuine Endigungsformel ist und in den Systematiken des 17. und 18. Jahrhunderts sogar als deren stärkste Vertreterin gehandelt wird, findet sie sowohl als Initialformel als auch als Sequenzbaustein Verwendung. Solange die Bassklausel zu Grunde liegt, lässt sich die quarta consonans, gefolgt vom Quart-Quint-Klang, als ihr konsitituierendes Moment begreifen. Bei Bassdiminution kann anstelle der quarta consonans ein konsonanter Klang treten, der Quart-Quint-Klang bleibt aber erhalten. Werden die Klauseln jedoch dergestalt getauscht, dass Diskantoder Tenorklausel im Bass stehen, zeigt sich die Verwandtschaft nur noch in den spezifischen melodischen Klauseln. Durch die Techniken des Klauseltauschs und der kontrapunktischen Kombinatorik wird es möglich, den gesamten Kontrapunkt mit cadenze doppie gleichsam zu durchsetzen. Daraus resultiert in der Regel eine Harmonik, die mit Hilfe der Oktavregel - und damit als «Kadenzharmonikı - beschrieben werden kann.

Während die Kadenzformel somit in Dur-Moll-tonaler Harmonik des 18. und 19. Jahrhunderts einerseits aufgehoben zu sein scheint, lebt sie in ihren ursprünglichen Formen, also fundiert durch die Bassklausel, als feste Formel auch im 19. Jahrhundert weiter. Der ihr ungeachtet ihrer Beliebtheit und ihres Entwicklungspotenzials im 17. Jahrhundert bereits von Vicentino zugeschriebene Charakter des Altmodischen oder Archaischen 
wird ihr im 19. Jahrhundert wieder zu Eigen. Daher dient sie gerade sromantischen ponisten als häufig verwendetes Versatzstück. Wie das Beispiel aus Wagners Tristan und Isolde illustriert, geht dabei von der Faszination, die die cadenza doppia aufgrund ihres Variantenreichtums und der damit einhergehenden Potenzialität in den verschiedensten Kontexten auf die Komponisten ausgeübt hat, nichts verloren. Die Präsenz bzw. Absenz der cadenza doppia in Lehrbüchern sowie ihrer Erscheinungsformen in der Musik des 19. und 20. Jahrhunderts wäre eine eigene Untersuchung wert.

\section{Literatur}

\section{Quellen}

Berardi, Angelo (1687), Documenti armonici, Bologna, Reprint Bologna: Forni 1970 (= BMB II/40a).

Bismantova, Bartolomeo (1677), Compendio Musicale, Ferrara, Reprint Florenz: Studio per edizioni scelte 1983.

Bononcini, Giovanni Maria (1673): Musico prattico, Bologna, Reprint Hildesheim u.a.: Olms 1969.

Dandrieu, Jean François (1719), Principes de l'accompagnement du clavecin, Paris, Reprint Genève: Minkoff 1972.

Durante, Francesco (2003), Bassi e Fughe, Ms. Napoli, Reprint Padua: Armelin Musica.

Fenaroli, Fedele (1775), Regole musicali per i principianti di cembalo, Napoli, Reprint Bologna: Forni 1975.

Furno, Giovanni (ca. 1817), Metodo facile breve e chiaro delle prime ed essensiali regole per accompagnare Partimenti senza numeri.

Gasparini, Francesco (1722), L'armonico pratico al cimbalo, Bologna, Reprint Bologna: Forni 2005.

Muffat, Georg (1699), Regulae Concentuum Partiturae.

Murschhauser, Franz Xaver (1721), Academia musico-poetica bipartita, Nürnberg: Endters.

Niedt, Friedrich Erhard (1710-1721), Musicalische Handleitung, Hamburg, Reprint Hildesheim u. a.: Olms 2003.

Ornithoparcus, Andreas (1517): Musice active micrologus, Leipzig.

Paisiello, Giovanni, (1782), Regole per bene accompagnare il partimento, Ms. Napoli, hg. von Ludwig Holtmeier, Johannes Menke und Felix Diergarten, Wilhelmshaven: Noetzel 2008.

Penna, Lorenzo (1684), Li primi albori musicali, Bologna, Reprint Bologna: Forni 1996.

Playford, John (1697), An Introduction to the Skill of Musick, 13. Auflage, London.

Tinctoris, Johannes (1477), Liber de arte contrapuncti. 
Tritto, Giacomo (1816), Scuola di Contrappunto, Napoli.

Vicentino, Nicola (1555), L'antica musica ridotta alla moderna prattica, Rom.

Walther, Johann Gottfried (1732), Musicalisches Lexicon, Leipzig, Reprint Kassel: Bärenreiter 1993.

Zarlino, Gioseffo (1558), Le istitutioni harmoniche, Venedig.

\section{Literatur}

Deppert, Heinrich (1993), Kadenz und Klausel in der Musik von J. S. Bach. Studien zu Harmonie und Tonart, Tutzing: Schneider.

Diergarten, Felix (2011), „Romantic Thoroughbass. Music Theory between Improvisation, Composition and Performance», Theoria. Historical Aspects of Music Theory 18, 7-36.

Dodds, Michael R. (2006), „Columbus's Egg: Andreas Werckmeister's Teachings on Contrapuntal Improvisation in ıHarmonologia musica (1702) «, Journal of SeventeenthCentury Music 12/1. http://sscm-jscm.press.uiuc.edu/v12/no1/dodds.html

Froebe, Folker (2009), "Satzmodelle des >Contrapunto alla mente für den Stilwandel um 1600«, ZGMTH 4/1-2, Hildesheim u. a.: Olms, 13-56.

Helmberger, Andreas und Ulrich Kaiser (o.J.), Artikel »Motivo di Cadenza", in: Musiktheorie aktuell. Online-Publikation: http://www.musiktheorie-aktuell.de/tutorials/ motivodicadenza.aspx.

Holtmeier, Ludwig (2010), Artikel »Kadenz/Klausel«, in: Lexikon der Systematischen Musikwissenschaft, hg. von Helga de la Motte Haber u. a., Laaber: Laaber 2010, 202-206.

— (2008), „Zum Tonalitätsbegriff der Oktavregel«, in: Systeme der Musiktheorie, hg. von Clemens Kühn und John Leigh, Dresden: Sandstein, 7-19.

Jans, Markus (1987), »Alle gegen Eine. Satzmodelle in Note-gegen-Note-Sätzen des 16. und 17. Jahrhunderts", in: Basler Jahrbuch für historische Musikpraxis 10, Winterthur: Amadeus, 101-120.

Menke, Johannes (2009), »Historisch-systematische Überlegungen zur Sequenz seit 1600«, in: Passagen (musik.theorien der gegenwart 3), hg. von Christian Utz und Martin Zenck, Saarbrücken: Pfau, 87-111.

Schwab-Felisch, Oliver (2010), Artikel »Satzmodell«, in: Lexikon der Systematischen Musikwissenschaft, hg. von Helga de la Motte Haber u. a., Laaber: Laaber, 415-419.

Weber, Hans Peter (2004), Generalbass-Compendium, 4. Aufl., Basel: Ms. im Eigendruck. 